\title{
Research on the Present Situation and Development of 3D Reconstruction Software Based on Image
}

\author{
Shenglan Dong ${ }^{1, a}$, Hongwei Zhang ${ }^{1, b}$ \\ Ordnance Engineering College, Shijiazhuang 050003, China \\ a429405235@qq.com, b
}

Keywords: image;3D reconstruction; software.

\begin{abstract}
With the development of 3D reconstruction software based on image, the number of this software is increasing, also they are different in theory. This article makes a brief introduction about 20 kinds of 3D reconstruction software based on image, and made a simple description and division.
\end{abstract}

\section{Introduction}

Three-dimensional reconstruction technology has been developed over 30 years, it is an important field of computer vision. In the field of medicine, heritage, architecture, e-commerce is widely used. Based on a typical three-dimensional image reconstruction method generally comprises [1]: image acquisition, camera calibration, image matching, 3D reconstruction and other steps. Three-dimensional reconstruction can be divided into four main ways: (1) based on the image; (2) using a probe or laser reader by-point access to data; (3) CT scan three-dimensional objects; and (4) three-dimensional optical scanner. Where in the three-dimensional reconstruction of image based on just a digital camera, simple operation, widely used.

\section{Software Review}

Image based on three-dimensional reconstructed image is to use the camera on your computer to reproduce three-dimensional objects. Depending on the number of the image can be divided into single three-dimensional image reconstruction and two or more images for three-dimensional reconstruction based on. Where in the three-dimensional reconstruction based on single image of simple, fast, less investment, reconstruction time is shorter, its reconstruction methods can be divided into three categories: (1) based on a large database; (2) based on the shape recovery techniques and traditional SFS method, this technique requires high light and gray and can not be achieved for curved objects unique reconstruction; (3) based on the image of the geometric projection information, this method of reconstruction low accuracy. There are many scholars dedicated to research-based three-dimensional reconstruction of a single image, in order to improve its accuracy. Based on two or more images of complex three-dimensional reconstruction of the operation, computationally intensive and costly reconstruction, but eventually come to better effect, the reconstruction method can be divided into three categories [2]: (1) stereoscopic method; (2) the moving image sequence method; (3) photometric stereo school. Many existing mature software, but it is difficult for simple classification, the following is a detailed description of these software:

\subsection{Cloud Reconstruction}

There Reconstruction Autodesk cloud-based software 123D Catch [3] and Photofly, Beijing unlimited world's Infinite 3D-Real Scenes / Objects, OM3D created by the Carnegie Mellon University, Smoothie-3D, North Branch Everbright commercial launch photo modeling platform services Hypr 3D and 3D Cloud and Microsoft developed Photosynth. The basic principle is that several software by multiple photos uploaded to the cloud over the network, the corresponding treatment in the cloud, and the cloud processing software in principle that several yet different. It can be downloaded from the clouds After a few minutes to a few minutes of waiting to the corresponding three-dimensional model. This method is downloaded to a higher model in general 
resolution, clarity is good. In which several software, Infinite 3D-Real Objects to be combined with other three-dimensional modeling software processing the bottom of the object. Hypr 3D applications there are still some problems. Photosynth ceased development in July 2015. OM3D is free and open source software, the use of single three-dimensional image reconstruction, can be directly converted into a three-dimensional picture of the object model, and its model is steerable and mobile. However, this functionality depends on the image and 3D model library on the Internet. Simply means that the model library model objects and photos compare and match, and finally to create three-dimensional models. Smoothie-3D is a photograph by block outline the contours of the object model of the object can be obtained, and can adjust the contour of the object that is the contour shape of the object. The operation is relatively simple. Greater impact on the operator of the three-dimensional model of precision.

In addition, Trimensional the Iphone is a app, in the dark with the lights illuminate the face can be a three-dimensional face model, 3D model resulting quality is not high. Application limitations.

\subsection{Offline software}

Better software available offline are Agisofa Photoscan, Realviz company ImageModeler, EOS company PhotoModeler, 3DSOM, Smart 3D Capture and Visual SFM binding PMVS / CMVS. That several existing commercial software is offline modeling software, the higher the cost of most of the software, the use of relatively simple, just an ordinary digital camera, with a multi-angle shoot multiple photos into the software, can be carried out automatically three-dimensional reconstruction. This is due to the different production of several software companies, each company has its own core technology, so there are some gaps in the modeling speed. PhotoModeler which includes two versions --PhotoModeler Pro and PhotoModeler Scanner, the latter is more a function of the high-density surface modeling module, and better mapping of irregular shape, and reduce the workload mark. Visualsfm structure from motion is carried out using three-dimensional reconstruction of the interface, it can only get sparse point cloud, must comply with the PMVS / CMVS use, obtain dense point cloud. Bundler is written by Dr. Noah Snavely SFM toolkit that can generate three-dimensional information is sparse points by multiple photos, belong to the category of SFM.

\subsection{Other off line software}

3DMeNow is a powerful three-dimensional portraits modeling software, as long as the two figures given image, and select the key point, a person is able to build a three-dimensional avatar model. And 3DMeNow also based on the input voice files to synthesize facial expressions and voice. At the same time it can be used with the deformation characteristics, facial expressions and lips and teeth goal mouth shapes. Morph target is to have multiple 3D model fusion deformation, the final synthesis of animation techniques. Its output model can be directly imported today's popular three-dimensional modeling software, so you can achieve broadcast-quality rendering and animation output. Simple to use. In 2005 biovirtual has released 3DMeNow Professional 2.

Insight 3D is a free open-source software, through a few pictures to determine the position of the object point, the formation of point cloud thus forming surface, and finally be able to stick to the surface of the corresponding photograph.

Canoma ${ }^{[4]}$ launched by Metacreations company. By the apex body of one set, according to the set of vertex modeling. Its shortcomings 1.0 version does not support the model framework circular sphere. Now only produce some simple shape model of objects, such as tables and chairs and other objects.

Just one 3-Sweep photo modeling needed. 3-Sweep by Tsinghua University and Tel Aviv University scientists have developed, this software allows users to click the mouse to extract objects in the photo, and edit it. Support for modeling objects need to be broadly understood as cubes, cylinders, balls and other objects, which limits the software only to man-made object modeling. 


\section{3 .The development of three-dimensional image reconstruction software}

\subsection{Accuracy needs to be improved}

Because based on the use of three-dimensional image reconstruction software more widely, all walks of life to its increasingly demanding. Precision three-dimensional model has become a very important issue. Cloud-based model accuracy using good, but when the model is not suitable to do when uploading to the cloud model, offline modeling software more suitable. So offline reconstructed three-dimensional modeling software model has been more and more attention.

\subsection{Photographing conditions to be reduced}

Most of the existing software for the camera to take the picture has certain requirements, modeling the effect of light and the object distance and the pixel photos can largely influence the object. Some software but there is a dedicated camera system. For the surface is not reflective, opaque objects most modeling software reconstructed model effect is not bad, but for the reflective surface is stronger, more transparent objects, most of the software indicates that modeling results needs to be improved. Such objects using cloud software or directly from the photo "extract" the software objects or can be successfully modeled.

\subsection{Rebuild time should be sped up}

For higher real-time modeling requirements, the object of a long time to achieve a three-dimensional reconstruction becomes a critical factor. This study further contribution depends on experts and scholars of the three-dimensional image reconstruction.

\subsection{Automated software operation}

Simplification and automation software operations to better advance the field of development to more three-dimensional image reconstruction based software. Allow more non-professionals to spend this kind of software.

\section{Summary and Outlook}

With the development of three-dimensional reconstruction technology, many of the scholars are based on three-dimensional image reconstruction problems in the algorithm method done a lot of research. Rapid development is also based on a three-dimensional image reconstruction software. These software under certain conditions has been able to successfully reconstruct the object. It has been widely used in some areas. Scholars to be a good research can be applied to software up to promote the development of more areas to image-based three-dimensional reconstruction software.

\section{Reference}

[1] Deng Yanzai three-dimensional computer reconstruction of image-based research [D] Xi'an: Xi'an University of Architecture and Technology .2011

[2] Sun Yuyang D technology review based on a single image reconstruction [J] North China University of Technology, 2011,23 (1): 9.

[3] The publisher is unknown. Autodesk 123D series expansion product. [J]. Television production .2012.

[4] David Em. Metacreations Canoma_ to 3D objects from images. [J]. Electronics and Computer .1999. 